\title{
SOME BASIC ELEMENTS TO ACHIEVE A FUTURE 1D SIMULATION OF WAVE PROPAGATION IN I.C.E. PIPES
}

\author{
David CHALET \\ Pascal CHESSE \\ Jean-François HETET \\ Xavier TAUZIA \\ Laboratory of fluid mechanics - U.M.R. C.N.R.S. 6598 \\ Ecole Centrale de Nantes \\ Nantes, FRANCE
}

\section{ABSTRACT}

The assumption of one-dimensional unsteady flows in the inlet and exhaust systems of turbocharged diesel engines is widely used although multi-dimensional simulations using fluid dynamics are also possible. However, difficulties persist concerning the boundary conditions, particularly at the pipe ends (inflow or outflow) and at the intra-pipe boundary conditions (sudden or gradual area changes, bends, junctions, etc.). This paper focuses on the two first steps leading to a 1D flow simulation code: the selection of a numerical scheme and the study of an open end boundary condition. The first section compares several numerical algorithms, including Lax-Wendoff, Flux-CorrectedTransport methods (FCT), and Harten-Lax-Leer (Riemann solver), extended to the second order. The selection criterion is the best compromise between numerical instabilities and computational time. A numerical study using the Fluent CFD code is then presented on a constant area duct in order to determine some characteristics at the pipe end, specifically the dead zone length and the throat area. Finally, a model parameterized by the pressure ratio between inlet and outlet is proposed.

\section{NOMENCLATURE}

\begin{tabular}{|c|c|l|}
\hline Variable & Unit & \multicolumn{1}{|c|}{ Description } \\
\hline$a_{1}$ & $\mathrm{~s}^{-2}$ & law coefficient \\
$\mathrm{a}_{2}$ & $\mathrm{~s}^{-2}$ & law coefficient \\
$\mathrm{a}_{\mathrm{s}}$ & $\mathrm{m} / \mathrm{s}$ & speed of sound \\
$\mathrm{a}_{\mathrm{si}}$ & $\mathrm{m} / \mathrm{s}$ & speed of sound at point $\mathrm{i}$ \\
$\mathrm{a}_{\text {sout }}$ & $\mathrm{m} / \mathrm{s}$ & outside speed of sound \\
$\mathrm{b}_{1}$ & $\mathrm{~s}^{-1}$ & law coefficient \\
$\mathrm{b}_{2}$ & $\mathrm{~s}^{-1}$ & law coefficient \\
$\mathrm{c}$ & $\mathrm{m} / \mathrm{s}$ & velocity of shock \\
$\mathrm{c}_{1}$ & - & law coefficient \\
$\mathrm{CFL}$ & - & criterion of Courant, Friedrichs, and Lewy \\
$\mathrm{D}$ & $\mathrm{m}$ & diameter of tube \\
$\mathrm{e}$ & $\mathrm{J} / \mathrm{kg}$ & specific internal energy
\end{tabular}

\begin{tabular}{|c|c|l|}
$\mathrm{F}$ & - & flux vector \\
$\mathrm{Ft}$ & $\mathrm{m}^{2}$ & throat area \\
$\mathrm{F}_{\mathrm{tube}}$ & $\mathrm{m}^{2}$ & cross section area \\
$\mathrm{G}$ & $\mathrm{m} / \mathrm{s}^{2}$ & friction term \\
$\mathrm{Lt}$ & $\mathrm{m}$ & dead zone length \\
$\mathrm{L}_{\text {tube }}$ & $\mathrm{m}$ & tube length \\
$\mathrm{Ma}$ & - & Mach number \\
$\mathrm{P}$ & $\mathrm{Pa}$ & Pressure \\
$\mathrm{P}_{\mathrm{init}}$ & $\mathrm{Pa}$ & initial tube pressure \\
$\mathrm{Pi}$ & $\mathrm{Pa}$ & pressure at point $\mathrm{i}$ \\
$\mathrm{Pout}$ & $\mathrm{Pa}$ & outside pressure \\
$\mathrm{q}$ & $\mathrm{J} / \mathrm{kg}$ & heat transfer term \\
$\mathrm{S}$ & - & source vector \\
$\mathrm{t}$ & $\mathrm{s}$ & Time \\
$\mathrm{t}_{1}$ & $\mathrm{~s}$ & analytical solution time \\
$\mathrm{t}_{2}$ & $\mathrm{~s}$ & 2nalytical solution time \\
$\mathrm{T}_{\mathrm{i}}$ & $\mathrm{K}$ & temperature at point $\mathrm{i}$ \\
$\mathrm{u}$ & $\mathrm{m} / \mathrm{s}$ & axial velocity \\
$\mathrm{u}_{\mathrm{i}}$ & $\mathrm{m} / \mathrm{s}$ & axial velocity at point $\mathrm{i}$ \\
$\mathrm{W}$ & - & flow state vector \\
$\mathrm{x}$ & $\mathrm{m}$ & axial coordinate \\
$\mathrm{x}_{\mathrm{sensor}}$ & $\mathrm{m}$ & sensor position \\
$\mathrm{y}$ & $\mathrm{m}$ & radial coordinate \\
$\gamma$ & - & ratio of specific heats \\
$\Phi\left(\theta_{\mathrm{i}}\right)$ & - & Flux limiter \\
$\theta_{\mathrm{i}}$ & - & ratio of consecutive gradients \\
$\rho$ & $\mathrm{kg} / \mathrm{m}^{3}$ & density \\
$\rho_{\mathrm{i}}$ & $\mathrm{kg} / \mathrm{m}^{3}$ & density at point $\mathrm{i}$ \\
& & \\
\hline
\end{tabular}

\section{INTRODUCTION}

For several decades, the inlet and exhaust systems of internal combustion engines have been the focus of numerous studies involving gas dynamics. The dimensions of these systems have a direct influence on the cylinder filling and emptying (in terms of average value and distribution among the cylinders), and thus on the combustion process, 
the overall engine performance and the pollutant emissions at the engine exhaust.

The first flow simulations were performed using the method of characteristics (Benson 1982). The ever-increasing performance of computers allowed for the advent of new resolution techniques resulting in numerous new algorithms (Chen and al. 1992, Corberán and al. 1995, Vandervoorde and al. 2000, Winterbone and al. 2000).

However, the modeling of the various singularities presented by the inlet and exhaust systems of internal combustion engines still remains challenging. In particular, the pressure losses associated with these singularities have been evaluated at steady state (Blair 1995) to simplify both the computations and experimental measurements. Although this technique brings satisfactory results, it does not take into account the wave distortion resulting from the unsteady nature of the flow through the singularity (for example, the change in position of the dead zones as a function of time).

The final objective of this study is to create a ID simulation code dedicated to Internal Combustion Engine. The first step is the choice of a numerical scheme to solve the gas dynamics equations for different types of pipe junctions (parts 1 and 2). The second step is the study of various singularities in unsteady flow conditions using the Fluent CFD as a numerical test bench. In this paper, the study is presented for a fully open end boundary condition (part 3 ). A same study will be, in the future, conducted for each type of singularity and (as a further development) then integrated in a ID simulation code.

The proposed solutions aim at increasing the capabilities of the SELENDIA simulation code that has been developed by the authors for a decade (Hétet and al. 1994, Hétet and al. 2000) by adding the modeling of pressure wave propagation.

\section{GOVERNING EQUATIONS}

The flow through the pipes joining the various singularities is assumed to be quasi one-dimensional. Although unsteady flows are rarely one-dimensional, experience shows that a one-dimensional approach is sufficient in most cases (Benson 1982). This assumption results in the longitudinal variation of the pipe cross section area. The properties of the flow through this pipe are uniform in each cross section (Esfandiari 1985).

The gas dynamics of one-dimensional flows are described by the following three equations: the continuity equation, the momentum equation and the energy conservation equation. This system of three equations can take the following form, known as "conservative:"

$$
\frac{\partial W}{\partial t}+\frac{\partial F(W)}{\partial x}=S
$$

$$
\begin{aligned}
& \text { Where: } \\
& W=\left[\begin{array}{c}
\rho \\
\rho u \\
\rho \cdot\left(e+\frac{u^{2}}{2}\right)
\end{array}\right]
\end{aligned}
$$

$$
\begin{aligned}
& F(W)=\left[\begin{array}{c}
\rho u \\
P+\rho u^{2} \\
\left(e+\frac{u^{2}}{2}+\frac{P}{\rho}\right) \cdot \rho u
\end{array}\right] \\
& S=\left[\begin{array}{c}
-\frac{\rho u}{F_{\text {tube }}} \cdot \frac{d F_{\text {tube }}}{d x} \\
-\frac{\rho u^{2}}{F_{\text {tube }}} \cdot \frac{d F_{\text {tube }}}{d x}-\rho G \\
-\left(\rho \frac{u^{2}}{2}+P \frac{\gamma}{\gamma-1}\right) \cdot \frac{u}{F_{\text {tubc }}}-\frac{d F_{\text {tube }}}{d x}+\rho q
\end{array}\right]
\end{aligned}
$$

\section{SELECTION OF A NUMERICAL SCHEME}

The modeling presented in this section supposes a perfect and non viscous fluid inside a constant section duct. The flow is assumed to be adiabatic. These assumptions presuppose that the $S$ vector is null.

Although analytical solutions can be established in some specific cases, solving equation (1.) requires the use of discretization methods. The proposed solution was obtained from an investigation performed on a 2 meter long pipe (with 1000 cells) assumed to be initially either depressurized (at .8 bar) or pressurized (at 1.5 bar). The various numerical schemes were compared with the analytical solution shown in Appendix A (derived at mid length of the pipe) with respect to precision and then among each other with respect to computational time.

Historically, the first technique that was used was the method of characteristics. It is based on the possibility to transform the set of equations with partial derivative terms into a set of equations with full derivative terms. However, even though this method allows for easy visualization of the pressure wave propagation, its major disadvantage relies in the fact that it can hardly be applied to the occurrence of a shock. As a result, this technique was not implemented by the authors.

The increasing performance of computers allowed for the use of techniques based on the resolution of finite differences with a second order precision. This led to the schemes of Lax-Wendroff (LW2), Mac Cormack (MC0 et MCI) and Rubin \& Burstein (RB) (Peyret 1977). However, implementation on our test case regarding an expansion wave with an initial pipe pressure of 1.5 bar as depicted in Figure 1 shows that this type of resolution brings numerical oscillations. This is due to the diffusive property of discretization errors. The results also show that the method of Mac Cormack is not suitable for this type of problem since the direction of the discretization (right or left) results in a better resolution for a given direction of the flow. Similar results are obtained in the case of shock waves, thus leading to the non selection of these methods by the authors. 


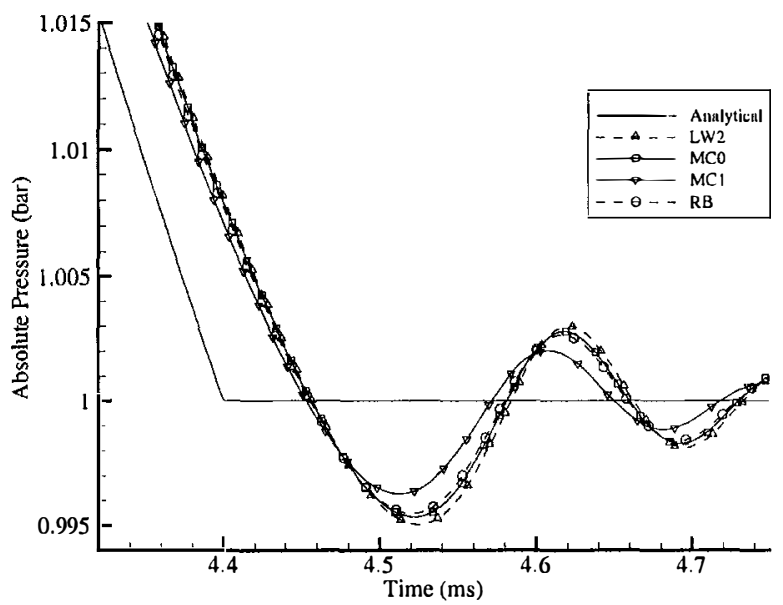

Figure 1. Comparison of resolution methods for the propagation of an expansion wave $(C F L=1.0)$ with an initial pressure of $1.5 \mathrm{bar}$

A first method was proposed to deal with numerical oscillations: the addition of an artificial viscosity in steady schemes (Peyret 1977). The anticipated objectives are a diffusive effect while preserving the precision, targeting zones with high gradients and preventing the shocks from spreading to a large number of points. The results are illustrated by Figure 2 in the case of shock waves. Similar results were obtained for each type of wave that was investigated. They show that this kind of model does not properly damp numerical oscillations.

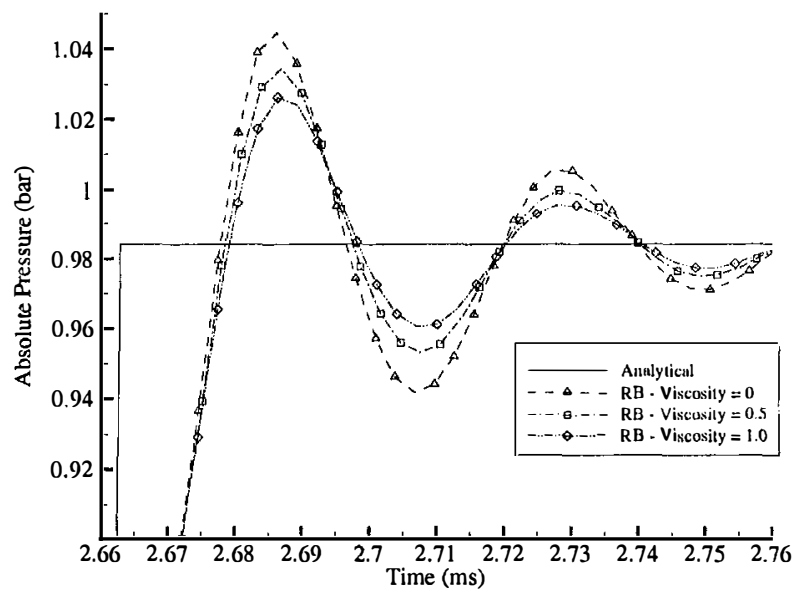

Figure 2. Influence of artificial viscosity on the resolution of a shock wave propagation ( $C F L=1.0$ ) with an initial pressure of

.8 bar

The addition of a diffusive term in the equations of the gas dynamics, rather than in the scheme itself, may also diminish numerical oscillations. This principle is known as artificial compression (Harten 1978). However, although this technique provides satisfactory results for basic flows, it is not suitable for complex flows such as those encountered inside pipes with variable sections (Bulaty and al. 1985).

The flux-corrected transport method (FCT) was then developed (Niessner and al. 1981). The methods based on this technique take advantage of first order schemes (monotone) as well as second order schemes (accurate). They rely on a weighted average of these two solutions. Investigation on shock and expansion waves by the authors shows that these resolution algorithms provide very satisfactory results. However, they lack the conservative properties used for the schemes of the Rubin \& Burstein class (Vandervoorde 2000). Consequently, they still generate a few numerical oscillations.

A solution that combines the use of the characteristic information with a conservative formulation was proposed by Godunov (Chen and al. 1992) in order to solve the problem by properly eliminating numerical instabilities. Instead of considering the characteristics at time $\mathrm{n}+\mathrm{l}$, Godunov suggested to solve Riemann's problems based on the characteristics at time $\mathrm{n}$. The approximated solution can then take the following form (while the source terms are still null):

$$
\mathrm{W}_{\mathrm{i}}^{\mathrm{n}+1}=\mathrm{W}_{\mathrm{i}}^{\mathrm{n}}-\frac{\Delta \mathrm{t}}{\Delta \mathrm{x}} \cdot\left(\mathrm{F}_{\mathrm{i}+\mathrm{y}_{2}}^{\mathrm{n}}-\mathrm{F}_{\mathrm{i}-1 / 2}^{\mathrm{n}}\right)
$$

Various numerical resolution schemes were then developed, particularly the scheme of Roe and the scheme of Harten-Lax-Leer, labeled HLL throughout this paper (Harten 1983). Roe's method consists of a local linearization at the $i+1 / 2$ interface followed by a full resolution of the problem. The considered element is weighted by density related terms starting from its boundaries (Roe's average) whereas the HLL technique uses an arithmetic average in conjunction with an explicit scheme of the first order, easily attainable.

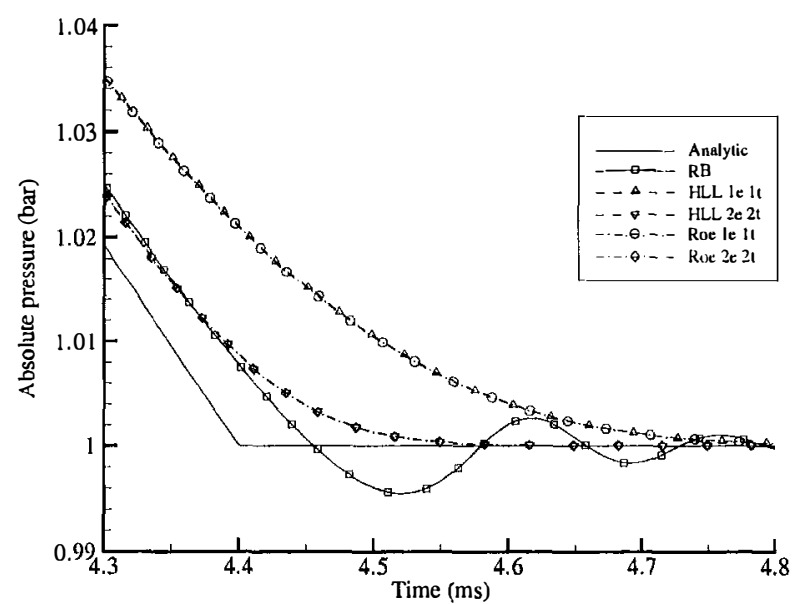

Figure 3. Comparison of resolution methods for the propagation of an expansion wave $(C F L=1.0)$ with an initial pressure of 1.5 bar

Figure 3, obtained from a comparative study involving an expansion wave, shows that numerical oscillations are no longer present; however, the convergence requires more time. As a result, this class of schemes is not as attractive as the class of Rubin \& Burstein due to the fact that Riemann's solvers are of the first order in terms of both time and space. The same conclusions can be drawn in the case of the propagation of a shock wave.

It thus appears that investigating higher orders of resolution becomes necessary. A total variation diminishing flux limiter algorithm (TVD) was used in this objective. 
The flux limiter $\Phi(\theta)$ of the numerical scheme can be selected among the following forms (Vandervoorde and al. 1998) where $\theta$ represents the ratio of consecutive gradients:

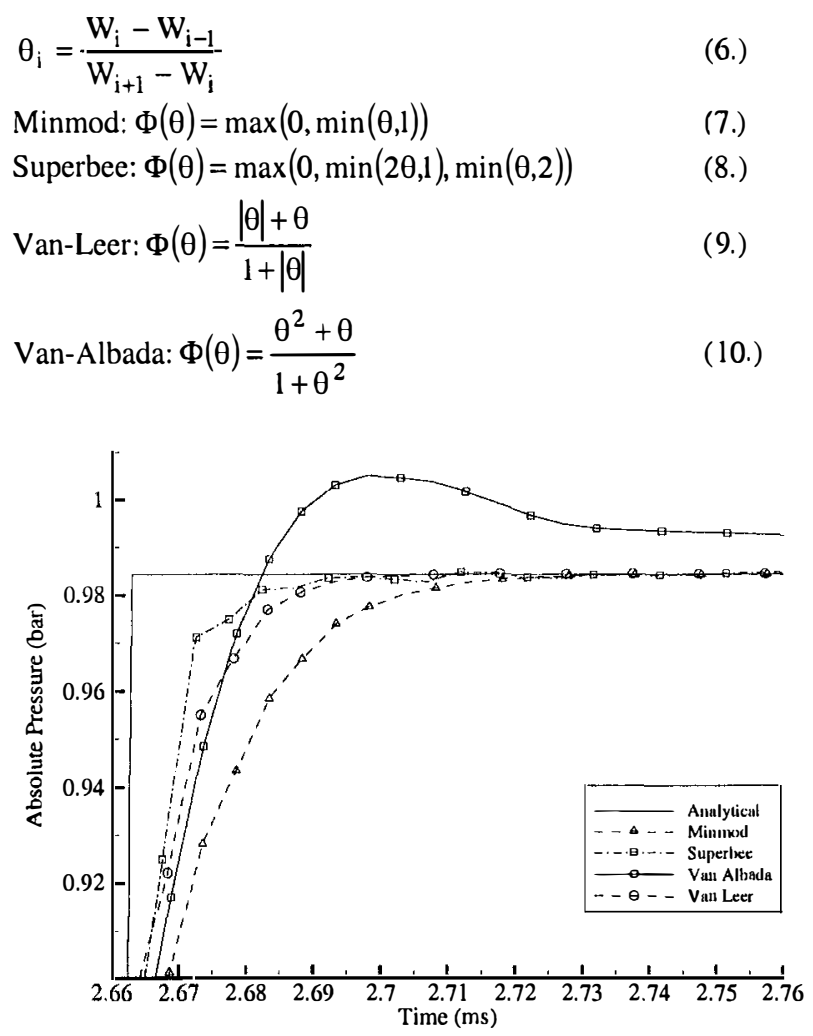

Figure 4. Selection of the flux limiter for the resolution of a shock wave propagation $(C F L=1.0)$ with an initial pressure of .8 bar

Figure 4 presents some results for the various flux limiters in the case of a shock wave propagation. It shows that the flux of Minmod and Van Albada are not as efficient as the flux of Van Leer.

Superbee's flux limiter shows a tendency to generate oscillations that do not diminish over time in the case of a shock wave. A sensitivity analysis of the CFL coefficient confirmed this trend. For this reason, the flux limiter of Van Leer provides the best results as far as solving the equations of the gas dynamics for the problem being investigated.

Switching to a second order resolution definitely improves the results. This is especially true for expansion waves as shown in Figure 3. The schemes of HLL and Roe are as accurate as the scheme of RB while presenting no numerical oscillations.

Figure 5 shows a comparison among the schemes in terms of computational time, using the LW 2 scheme for reference. It shows that Roe's scheme is very penalizing. As an example, the simulation of a $100 \mathrm{~ms}$ expansion wave propagation was performed using a pipe that comprises 1,000 cells. The computational time on a personal computer equipped with an $853 \mathrm{MHz}$ processor is 26.5 seconds for the LaxWendroff scheme whereas the Riemann solvers, of the second order in terms of both time and space, require 141.9 and 318.1 seconds for Harten-Lax-Leer and Roe, respectively.
Consequently, the method selected by the authors was the scheme of HLL with a second order reconstruction using a total variation diminishing Van Leer flux limiter algorithm.

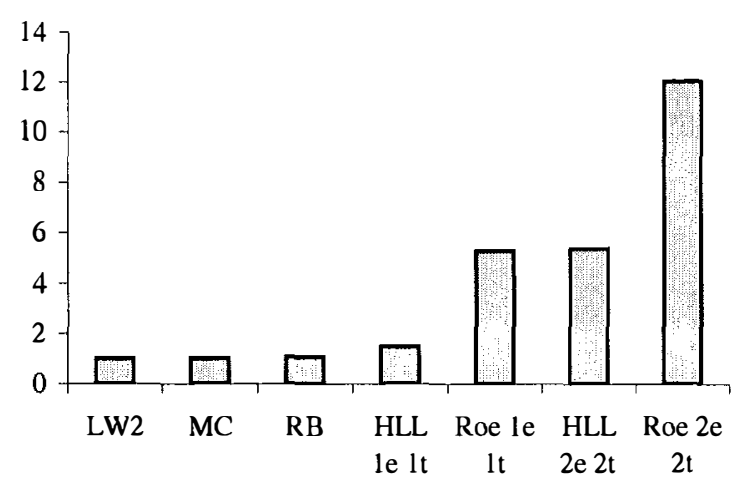

Figure 5. Relative computational times

\section{INVESTIGATION AND MODELING OF THE PLAIN OPEN END BOUNDARY CONDITION}

\subsection{Problem statement}

One-dimensional simulation must use adapted models since pipe inflows are not isentropic. The study presented in this paper does not involve a steady flow unlike most studies (Kirkpatrick 1994, Blair 1995, Fleck and al. 1996), but an unsteady flow to better characterize the plain open end boundary condition.

As a matter of fact, considering a pipe inflow from the atmosphere, it is possible to investigate the differences between the model selected by Kirpatrick (1D simulation) and the results given by Fluent and also by experimental measurements (see Figure 8).

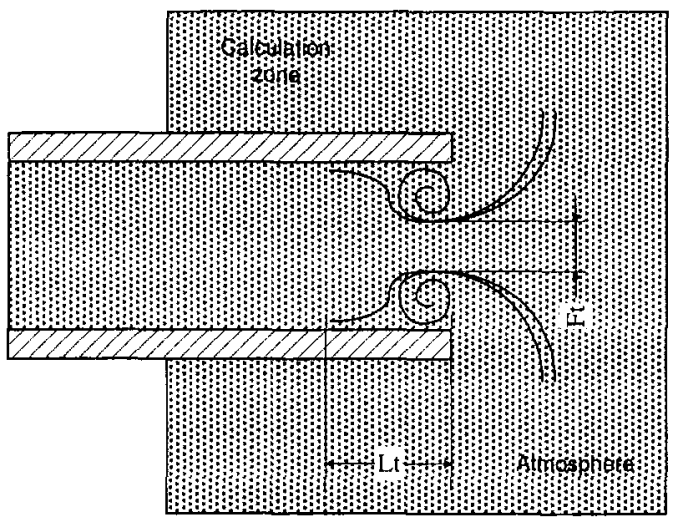

Figure 6. Flow penetrating from the outside

A 2D axisymmetric simulation was conducted using the FLUENT 5 CFD code in order to exhibit the losses generated by this type of singularity. Investigation of the inlet air boundary condition (considering a laminar viscous flow) was performed on a pipe with a $27 \mathrm{~mm}$ diameter and a $2.5 \mathrm{~mm}$ thickness. 

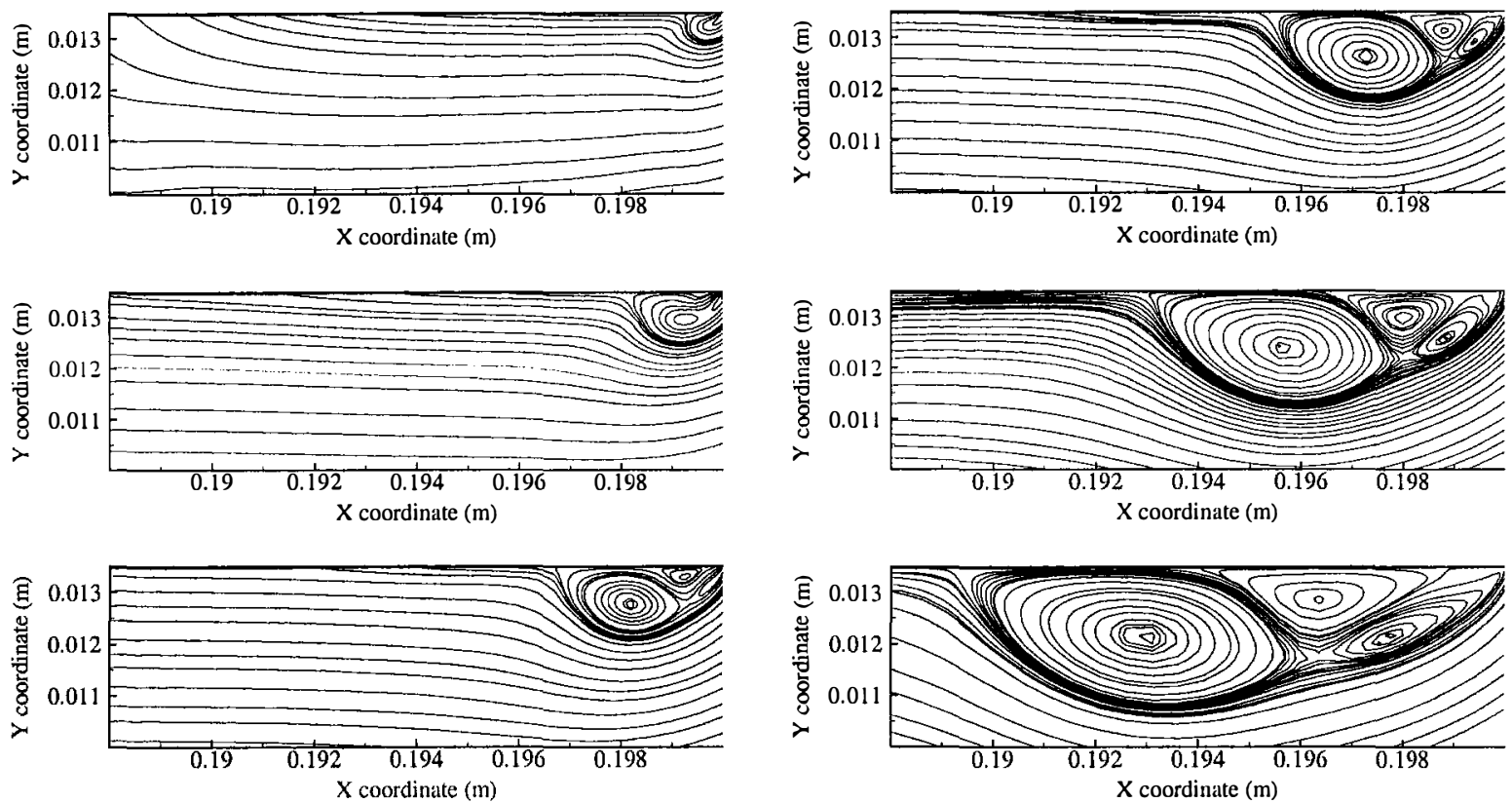

Figure 7. Stream function for a pipe inflow with an initial pressure of $.8 \mathrm{bar}$ at $\mathrm{t}=.03 \mathrm{~ms}, \mathrm{t}=.07 \mathrm{~ms}, \mathrm{t}=.13 \mathrm{~ms}, \mathrm{t}=.19 \mathrm{~ms}, \mathrm{t}=.3 \mathrm{~ms}, \mathrm{t}=.5 \mathrm{~ms}$

The pipe edges present a 90 degree angle as shown in Figure 6. The simulation process consists of allowing outside air to penetrate the pipe at $\mathrm{t}=0$ with an initial inner pressure of $\mathrm{P}_{\mathrm{inil}}$. Outside conditions are assumed to be 1 bar and $300 \mathrm{~K}$.

The grid used to model the pipe (27976 cells) is irregular. It is composed of smaller cells near the pipe walls with a first cell of .01 $\mathrm{mm}$ and a subsequent geometrical increase by a factor of 1.1 until the cells reach $1 \mathrm{~mm}$. The air inflow condition in Fluent assumes parallel streamlines. Actually, this assumption is not correct. The domain investigated is then the pipe itself and the atmospheric zone near the pipe entry using the same type of grid with 11096 cells (see Figure 6).

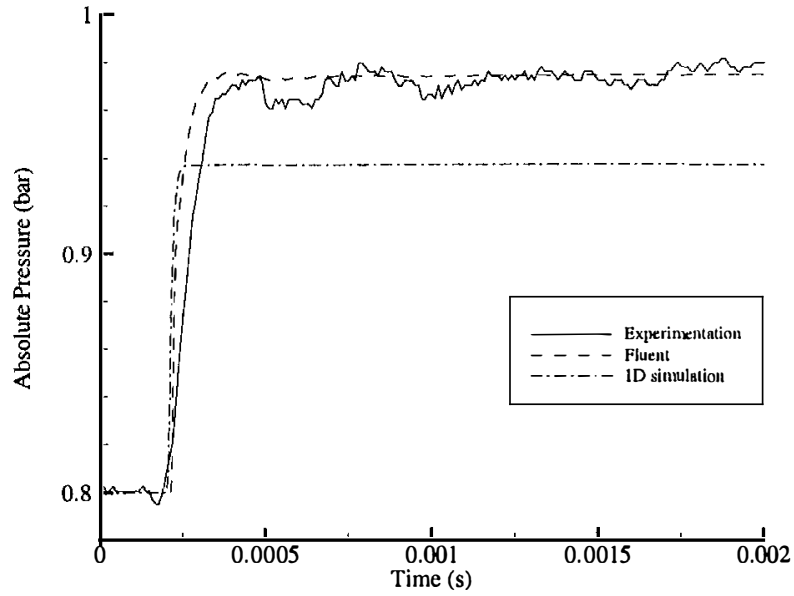

Figure 8. Comparison of pressure wave solutions near the plain open end boundary with an initial pressure of .8 bar
The simulation of the pipe filling (creation of a shock wave inside the pipe) shows the emergence of dead zones. Figures 7 and 9 obtained with an initial pressure of .8 bar show that the dead zones are not stationary. Their evolution is rapid. Furthermore, they heavily depend on the compression ratio.

One can also observe that the various geometrical characteristics of the dead zone are not constant. Figures 7 and 9 show that the fluid throat area $\mathrm{Ft}$ and the dead zone length $\mathrm{Lt}$, defined in Figure 6, keep varying as long as the flow is unsteady in this zone. Next part presents the graphical studies of $\mathrm{Ft}$ and $\mathrm{Lt}$ based on the streamline.

\subsection{Determination of geometrical characteristics related to the flow}

The initial focus was put on the throat area $\mathrm{Ft}$ to be graphically determined from the streamlines. In this objective, several simulation runs with different initial inner pressures $(.80, .85, .90$ and .95 bar) were conducted for a flow coming from the outside and going inside the pipe. Figure 10 shows $\mathrm{Ft}$ as a function of time.

The following model describes the variations of $\mathrm{Ft}$ as a function of time and initial pressure ratio:

$$
\frac{F_{t}}{F_{\text {tube }}}=\frac{a_{1}}{\left(\frac{t \cdot a_{\text {sout }}}{D}\right)+b_{1}}+c_{1}
$$

Where:

$a_{1}=b_{1} \cdot\left(1-c_{1}\right)$ 

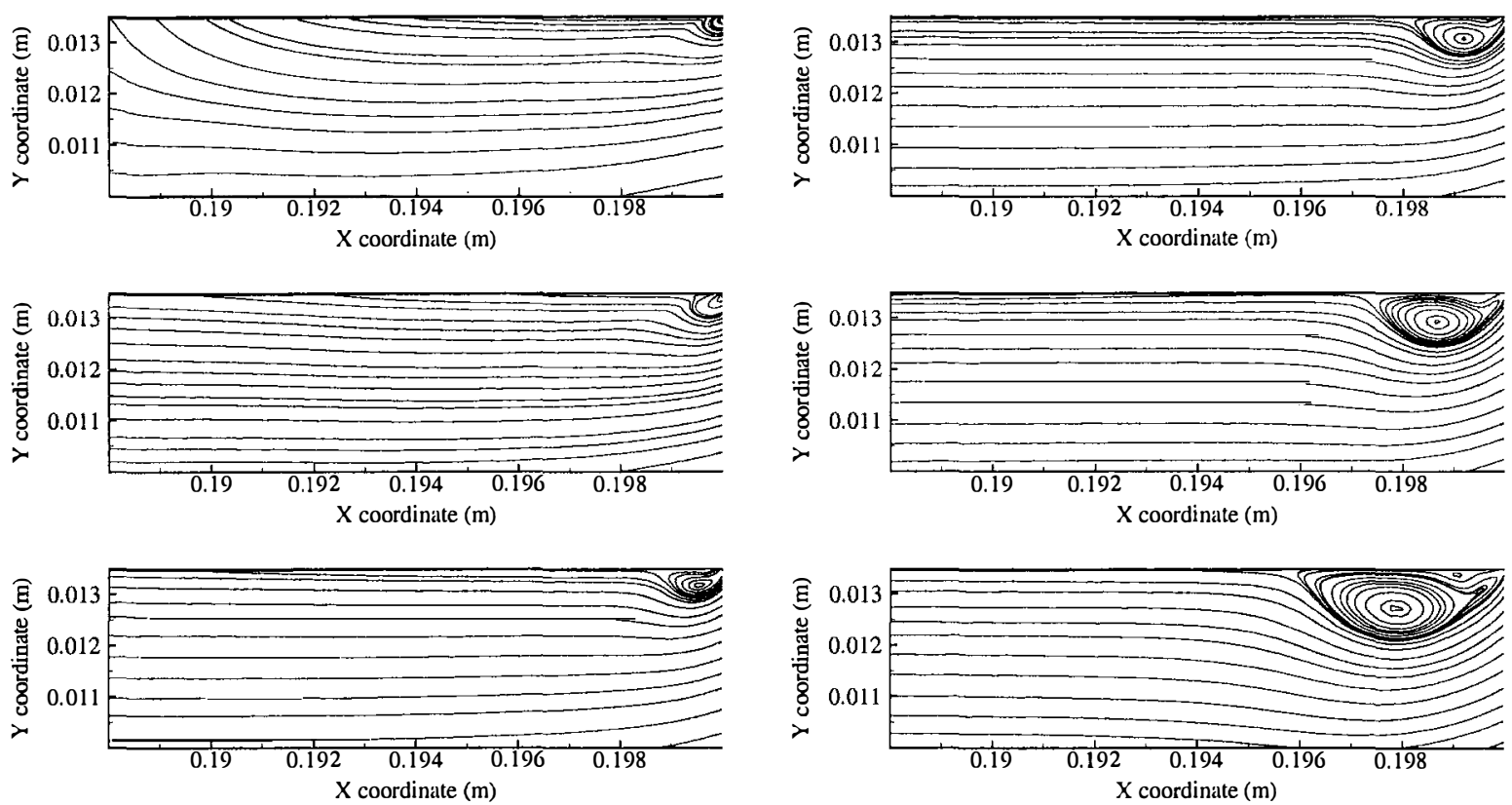

Figure 9. Stream function for a pipe inflow with an initial pressure of .95 bar at $\mathrm{t}=.03 \mathrm{~ms}, \mathrm{t}=.07 \mathrm{~ms}, \mathrm{t}=.13 \mathrm{~ms}, \mathrm{t}=.19 \mathrm{~ms}, \mathrm{t}=.3 \mathrm{~ms}, \mathrm{t}=.5 \mathrm{~ms}$

$$
\begin{aligned}
& \mathrm{b}_{\mathrm{l}}=-\frac{0.48}{\left(\frac{\mathrm{P}_{\text {init }}}{\mathrm{P}_{\text {out }}}\right)^{1.4}-1}+1.1 \\
& \mathrm{c}_{\mathrm{l}}=0.13 \cdot\left(\frac{\mathrm{P}_{\text {init }}}{\mathrm{P}_{\text {out }}}\right)^{8}+0.444
\end{aligned}
$$

Figure 10 shows that some of the data points are not properly evaluated by this model. However, the difference between the model and the FLUENT computations remains minor.

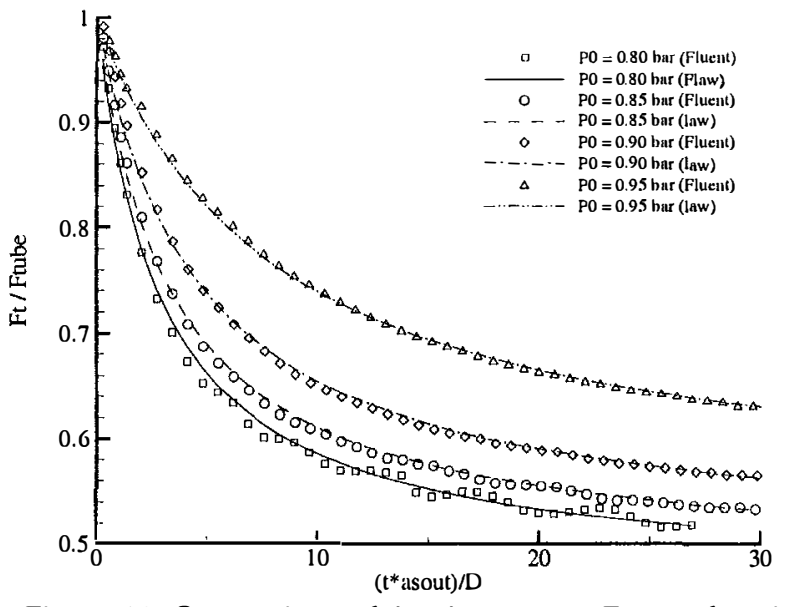

Figure 10. Comparison of the throat area $\mathrm{Ft}$ as a function of time and initial pressure
The simulation results presented above may also be used to evaluate the dead zone length, Lt (Figure 11). The following model taking the form of a logarithmic equation can be proposed:

$$
\frac{\mathrm{L}_{\mathrm{t}}}{\mathrm{D}}=\mathrm{a}_{2} \cdot \ln \left(1+\mathrm{b}_{2} \cdot\left(\frac{\mathrm{t} \cdot \mathrm{a}_{\text {sout }}}{\mathrm{D}}\right)\right)
$$

Where:

$$
\begin{aligned}
& \mathrm{a}_{2}=\frac{3.3}{\left(\frac{\mathrm{P}_{\text {init }}}{\mathrm{P}_{\text {out }}}\right)^{4.6}}-2.9 \\
& \mathrm{~b}_{2}=0.06 \cdot\left(\frac{\mathrm{P}_{\text {init }}}{\mathrm{P}_{\text {out }}}\right)-0.038
\end{aligned}
$$

The determination of Ft and Lt uses:

$$
\frac{\mathbf{P}_{\text {init }}}{\mathrm{P}_{\text {out }}}<1
$$

The results show that the throat area Ft and the dead zone length Lt are not time constant. While the throat area $\mathrm{Ft}$ varies, dead zones are created. The energy involved in this creation disturbs the pipe inflow. This energy is time dependant and as a consequence the loss coefficients used in boundary condition modeling are also time dependant. The knowledge of the length Lt leads us to locate where the 
flow is not 1D. Furthermore, it evaluates the energy necessary for the dead zones displacement after their occurrences.

As a result, it appears that a pressure loss coefficient model based on simulation and steady state measurements is not accurate enough for this type of boundary condition with respect to the application being investigated.

These results were established for a pipe identical to the pipe shown in Figure 6, i.e., with edges presenting a 90 degree angle. Further investigations are necessary to analyze the effect of curved edges to take into account the geometrical characteristics of the pipes that are typically used for the inlet and exhaust systems of internal combustion engines. Another area of further research includes investigating the influence of the pipe diameter and thickness on the various results.

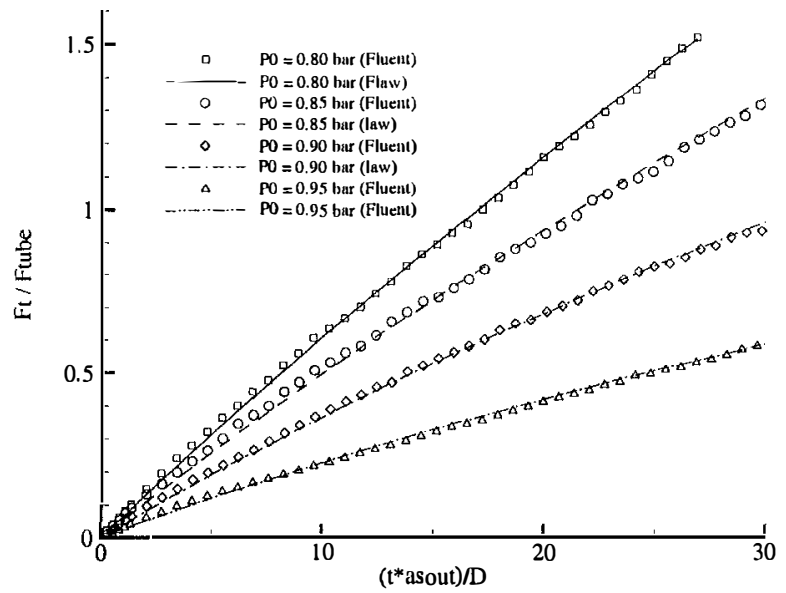

Figure 11. Comparison of the dead zone length $L t$ as a function of time and initial pressure

\section{CONCLUSION}

Modeling the propagation of pressure waves inside the piping systems of internal combustion engines first requires the selection of a numerical scheme to solve the equations of the gas dynamics. First order algorithms present the advantage of being monotone, simple and consequently cost effective in terms of computational time. Second order schemes in terms of both time and space are required to improve the model's accuracy. Using total variation diminishing flux limiter methods removes the oscillating nature of these algorithms. For the application being investigated, the best compromise with respect to accuracy and computational time is the scheme of Harten-Lax-Leer using a second order reconstruction by a total variation diminishing flux limiter method based on Van-Leer.

In addition, the one-dimensional modeling of pipe inflows requires certain geometrical characteristics. The proposed model for the fluid throat area will allow for the determination of the actual mass flow at the pipe inlet and consequently the establishment of a proper model for the losses inherent to this type of singularity; however, further investigations of the geometry of this inlet air element are needed to corroborate the results with actual cases.
A comparison with experimental results will be the subject of a follow-up project. An experimental setup is being developed. The objective will consist of investigating the effect of a pipe filling and emptying on the pipe inner pressure. Various geometrical configurations will be tested. The global results will then be integrated into the SELENDIA code for the simulation of turbocharged diesel engines.

\section{ACKNOWLEDGEMENTS}

We would like to thank the Centre National de la Recherche Scientifique and the Region des Pays de la Loire for their financial support.

\section{REFERENCES}

Benson R.S., Horlock J.H., Winterbone D.E., 1982, "The thermodynamics and gas dynamics of internal combustion engines", Vol. 1, Clarendon Press, Oxford.

Blair G.P., 1995, "Coefficients of discharge at the apertures of engines", SAE Transactions, Vol. 104, No. 3, pp. 2048-2062.

Bulaty T., Niessner H., 1985, "Calculation of 1-D unsteady flows in pipe systems of I.C. Engines", Journal of Fluids Engineering, Vol. 107, No. 2, pp. 407-412.

Chen C., Veshagh A., 1992, "A comparison between alternative methods for gas real flow and performance prediction of internal combustion engines", SAE Transactions, pp. 1793-1824.

Corberán J.M., Gascón L., 1995, "New method to calculate unsteady 1-D compressible flow in pipes with variable cross section. Application to the calculation of the flow in intake and exhaust pipes of I.C. Engines", ICE Engine Modeling ASME, Vol. 23, pp. 77-87.

Esfandiari C., 1985, "Simulation numérique des écoulements pulsés instationnaires dans les conduites avec singularités de section" PhD Thesis - Ecole Nationale Supérieure des Arts et Métiers de Paris.

Fleck R., Cartwright A., 1996, "Coefficients of discharge in high performance two-stroke engine", SAE Transactions, Vol. 105, No. 3, pp. 2469-2480.

Harten A., 1978, "The artificial compression method for computation of schocks and contact discontinuities: III. Self-adjusting hybrid schemes", Mathematics of computation, Vol. 32, No. 142, pp. 363-389.

Harten A., 1983, "High resolution schemes for hyperbolic conservation laws", Journal of Computational Physics, Vol. 49, pp. 357-393.

Hétet J.-F., Chessé P., Inozu B., 1994, "An ACSL simulation for optimum operation of turbocharged marine Diesel engines" ETCE'94 conference, ASME Internal Combustion Engine-Symposium, New Orleans, proceedings No. 94, ICE-7, pp. 1-13.

Hétet J.-F., Inozu B., Roy P., Tauzia X., Chessé P., 2000, "Performance simulation of sequentially turbocharged Marine Diesel Engines with applications to compressor surge" - Journal of Engineering for Gas Turbines and Power, Special ICE Issue, Vol. 122, No. 4, pp. 562-569.

Kirkpatrick S.J., 1994, "An evaluation of one-dimensinal simulation techniques for predicting unsteady gas flow in engine 
ducting", Ph D. Thesis, Department of Mechanical and Manufacturing Engineering, The Queen's University of Belfast.

Niessner H., Bulaty T., 1981, "A family of Flux-Correction Method to avoid overshoot occuring with solutions of unsteady flow problems", Proceedings of . GAMM 4th Conf., pp. 241-250.

Peyret R., 1977, "Résolution numérique des systèmes hyperboliques. Application à la dynamique des gaz", ONERA Publication, No. 1977-5.

Vandervoorde M., Vierendeels J., Dick E., Sierens R., 1998, "A new total variation diminushing scheme for the calculation of onedimensional flow in inlet and exhaust pipes of internal combustion engines", Proceedings of the Institute of Mechanical Engineers, Part D., Journal of Automobile Engineering, Vol. 212, No. 5, pp. 437-448.

Vandervoorde M., Vierendeels J., Sierens R., Dick E., Baert R., 2000, "Comparison of algorithms for unsteady flow calculations in inlet and exhaust systems of I.C. engines", Journal of Engineering for Gas Turbines and Power, Special ICE Issue,Vol. 122, No. 4, pp. 541548.

Winterbone D.E., Pearson R.J., 2000, "Theory of Engine Manifold Design - wave action methods for I.C. Engines", Professional Engineering Publishing Limited, London.

\section{APPENDIX A}

The initial objective is concerned with an analytical solution for the expansion wave propagation. It is assumed that there is no loss at the singularity. The method of characteristics provides the following direction equation and compatibility condition (Benson 1982):

$$
\begin{aligned}
& \frac{d x}{d t}=u-a_{s} \\
& a_{s}+\frac{\gamma-1}{2} \cdot u=a_{s 0}
\end{aligned}
$$

The proposed analytical solution provides the time variations of the pressure at a given point of the pipe $\left(\mathrm{x}_{\text {sensor }}\right)$. Equations (18.) and (19.) combined with the isentropic property of the flow lead to the following equation:

$$
\begin{aligned}
& a_{s}=\left[\frac{L_{\text {tube }}-x_{\text {sensor }}}{t}+\frac{2}{\gamma-1} \cdot a_{s 0}\right] \cdot \frac{\gamma-1}{\gamma+1} \\
& P=P_{0} \cdot\left(\frac{a_{s}}{a_{s 0}}\right)^{\frac{2 \cdot \gamma}{\gamma-1}}
\end{aligned}
$$

These two equations are valid between time $\left(t_{1}\right)$ at which the wave starts passing through the sensor and time $\left(\mathrm{t}_{2}\right)$ at which it ends. Their values are the following:

$$
\begin{aligned}
& \mathrm{t}_{1}=\frac{\mathrm{L}_{\text {tube }}-\mathrm{x}_{\text {sensor }}}{\mathrm{a}_{\mathrm{s} 0}} \\
& \mathrm{t}_{2}=(\gamma-1) \cdot \frac{\mathrm{L}_{\text {tube }}-\mathrm{x}_{\text {sensor }}}{(\gamma+1) \cdot \mathrm{a}_{\text {sout }}-2 \cdot \mathrm{a}_{\mathrm{s} 0}}
\end{aligned}
$$

A second objective is concerned with the shock wave propagation. The tluid inside the pipe is assumed to be divided into the following three distinct zones as shown in Figure A1:

- State 0: no fluid motion, initially in the pipe.

- State 1: fluid in motion, initially in the pipe.

- State 2: fluid in motion, initially outside.

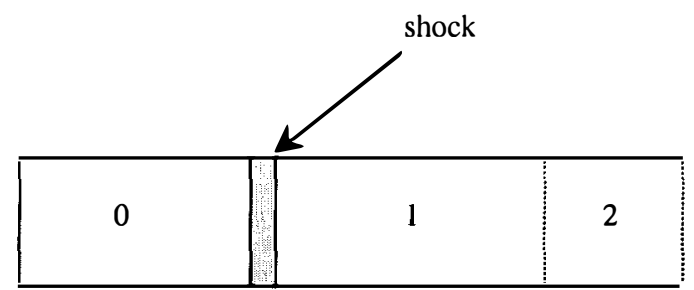

Figure A1. Fluid States

The shock wave separates the fluid into two entities whose thermodynamic variables are linked by the Rankine-Hugoniot relations as well as the shock continuity equation. It is assumed that there is no loss at the singularity, i.e., the flow is isentropic between the outside and the pipe inlet. This provides the energy conservation equation. The resulting equations combined with the definition of the shock propagation velocity, the sound celerity, and ideal gas state equation lead to the following set of equations:

$$
\begin{aligned}
& P_{2}=P_{1} \\
& \frac{P_{1}}{P_{0}}=1+\frac{2 \cdot \gamma}{\gamma+1} \cdot\left(M_{a}^{2}-1\right) \\
& \frac{\rho_{0}}{\rho l}=1-\frac{2}{\gamma+1} \cdot \frac{M_{a}^{2}-1}{M_{a}^{2}} \\
& \frac{P_{1}}{\rho_{1}}=r \cdot T_{1} \\
& a_{s 1}^{2}=\gamma \cdot r \cdot T_{1} \\
& P_{2}=P_{o u t} \cdot\left(\frac{a_{s 2}}{a_{\text {sout }}}\right)^{\frac{2 \cdot \gamma}{\gamma-1}} \\
& a_{s 2}^{2}+\frac{\gamma-2}{2} \cdot u_{2}^{2}=a_{\text {sout }}^{2} \\
& u_{1}=u_{2} \\
& a_{s 2}^{2}=\gamma \cdot r \cdot T_{2} \\
& \frac{P_{2}}{\rho_{2}}=r \cdot T_{2} \\
& c=M a \cdot a_{s} \\
& c=\frac{\rho_{1}}{\rho_{1}-\rho_{0}} \cdot u_{1}
\end{aligned}
$$

The resolution is performed based on an iterative procedure on $\mathrm{P} 1$. As an example, $P_{0}=.8$ bar leads to $P_{1}=.9843$ bar and a shock propagation velocity of $375.5 \mathrm{~m} . \mathrm{s}^{-1}$. 